\title{
Spectroscopy of the helium-rich binary ES Ceti reveals accretion via a disc and evidence of eclipses
}

\author{
K. Bạkowska ${ }^{1}$, T. R. Marsh ${ }^{2}$, D. Steeghs ${ }^{2}$, G. Nelemans ${ }^{3,4,5}$, and P. J. Groot ${ }^{3,6,7,8}$ \\ ${ }^{1}$ Institute of Astronomy, Faculty of Physics, Astronomy and Informatics, Nicolaus Copernicus University, ul. Grudziądzka 5, \\ 87-100 Toruń, Poland \\ e-mail: bakowska@umk.pl \\ 2 Department of Physics, University of Warwick, Coventry CV4 7AL, UK \\ 3 Department of Astrophysics/IMAPP, Radboud University, PO Box 9010, 6500 GL Nijmegen, The Netherlands \\ ${ }^{4}$ Institute of Astronomy, KU Leuven, Celestijnenlaan 200D, 3001 Leuven, Belgium \\ 5 SRON, Netherlands Institute for Space Research, Sorbonnelaan 2, 3584 CA Utrecht, The Netherlands \\ 6 Department of Astronomy, University of Cape Town, Private Bag X3, Rondebosch 7701, South Africa \\ 7 South African Astronomical Observatory, PO Box 9, Observatory 7935, South Africa \\ 8 The Inter-University Institute for Data Intensive Astronomy, University of Cape Town, Private Bag X3, Rondebosch 7701, \\ South Africa
}

Received 26 August 2020 / Accepted 18 November 2020

\begin{abstract}
Context. Amongst the hydrogen-deficient accreting binaries known as the AM CVn stars are three systems with the shortest known orbital periods: HM Cnc (321 s), V407 Vul (569 s), and ES Cet (620 s). These compact binaries are predicted to be strong sources of persistent gravitational wave radiation. HM Cnc and V407 Vul are undergoing direct impact accretion in which matter transferred from their donor hits the accreting white dwarfs directly. ES Cet has the longest period of the three and is amongst the most luminous AM CVn stars, but it is not known whether it accretes via a disc or direct impact. ES Cet displays strong HeII 4686 line emission, which is sometimes a sign of magnetically controlled accretion. Peculiarly, although around one third of hydrogen accreting white dwarfs show evidence of magnetism, none have been found amongst helium accretors.

Aims. We present the results of Magellan and VLT spectroscopic and spectropolarimetric observing campaigns dedicated to ES Cet with the aim of understanding its accretion structure.

Methods. Based on the data collected, we derived trailed spectra, computed Doppler maps of the emission lines, and looked for circular polarisation and variability.

Results. We find strong variability in our spectra on the 620 s period. The lines show evidence of double-peaked emission, characteristic of an accretion disc, with an additional component associated with the outermost disc, rather than a direct impact, which is broadly consistent with S-wave emission from the gas stream or disc impact region. This confirms beyond any doubt that $620 \mathrm{~s}$ is the orbital period of ES Cet. We find no significant circular polarisation (below 0.1\%). The trailed spectra show that ES Cet's outer disc is eclipsed by the mass donor, revealing at the same time that the photometric minimum coincides with the hitherto unrecognised eclipse.

Conclusions. ES Cet shows spectroscopic behaviour consistent with accretion via a disc, and is the shortest orbital-period eclipsing
\end{abstract} AM CVn star known.

Key words. binaries: close - white dwarfs - novae, cataclysmic variables - stars: individual: ES Ceti

\section{Introduction}

The AM Canum Venaticorum (AM CVn) stars are ultra-compact binary systems with orbital periods of 5-65 min in which white dwarfs accrete from degenerate or semi-degenerate companions (recent reviews in Solheim 2010; Ramsay et al. 2018). Only hydrogen-deficient material can attain the density necessary to fit within Roche lobes at such short orbital periods, and the spectra of AM CVn stars are devoid of hydrogen lines and are dominated instead by helium, sometimes along with with heavier elements (see Warner 1995 for a detailed review).

Cataclysmic variables (CVs) and AM CVn stars share many common features, including accretion discs that undergo semiregular cycles of outburst and quiescence in some cases, and features characteristic of these discs are seen in the spectra of both classes. There are also differences between the two types; obviously their abundances and orbital periods are very different, but two other distinctions can be drawn. As yet there are no known examples of magnetic white dwarfs amongst almost 60 known AM CVn stars, whereas around one third of CVs are magnetic (Pala et al. 2020); there is no obvious explanation for this difference. The other difference is that AM CVn stars can reach such short periods that a novel form of accretion becomes possible in which no accretion disc forms, but matter directly impacts the accreting white dwarf instead (Marsh \& Steeghs 2002; Marsh et al. 2004).

For decades following the recognition of the nature of AM CVn itself (Smak 1967), only a few members of the class were identified. Even by the year 2000, just six systems were known, but wide-field spectroscopic and photometric surveys have since had a significant impact. By the time of Solheim (2010), 25 systems were known, while 57 were listed 
by Ramsay et al. (2018). Because of their short orbital periods, the evolution of AM CVn stars is expected to be governed by gravitational-wave radiation (Paczyński 1967). The radiation emitted from this hydrogen-deficient class of objects should be detectable by instruments such as the Laser Interferometer Space Antenna (LISA), hence they are test-beds of gravitational wave physics (Kupfer et al. 2018). AM CVn stars are also of interest as a potential progenitor class of Type Ia supernovae (Brown et al. 2011; Gilfanov \& Bogdán 2010; Shen \& Bildsten 2014) and can offer important insights into binary evolution and common-envelope evolution.

Three channels of formation for AM CVn stars have been proposed. One possibility is via a double degenerate system that initially starts out as detached and then evolves to become interactive via mass transfer. However, this path has been in doubt since Shen (2015) found that it was vulnerable to mergers caused by classical nova-like eruptions from the accreting white dwarf. Another possibility is via a semi-degenerate helium (He) star channel, while a third is to evolve from a hydrogen-rich $\mathrm{CV}$ (see e.g., Nelemans et al. 2001a,b; Podsiadlowski et al. 2003; Yungelson 2008; Brooks et al. 2015). We currently face a conundrum in understanding the origin of helium-rich CVs, because all identified pathways to their formation face problems when confronted with observations: for example, the recent study of the eclipsing AM CVn, Gaia 14aae (Green et al. 2019).

The star ES Ceti (ES Cet) was discovered by Noguchi et al. (1980) and classified as a CV by Downes \& Shara (1993). The optical magnitude range of the star is 16.5-16.8, and with an absolute magnitude of $M_{g}=5.6 \pm 0.4$, ES Cet is amongst the most luminous AM CVn stars (Ramsay et al. 2018). Warner \& Woudt (2002) found a photometric period in optical photometry of ES Cet of $620 \mathrm{~s}$. Photometric studies over a baseline of years showed the period to be highly coherent, albeit slowly increasing with time (Espaillat et al. 2005; Copperwheat et al. 2011; de Miguel et al. 2018). Combined with the helium-dominated emission line spectrum, it was natural to interpret the period as orbital. ES Cet has the third shortest orbital period currently known amongst AM CVn stars, after HM Cnc $\left(P_{\text {orb }}=324 \mathrm{~s}\right.$, Roelofs et al. 2010) and V407 Vul $\left(P_{\text {orb }}=569 \mathrm{~s}\right.$, Haberl \& Motch 1995). ES Cet's period increases at a rate consistent with expectations for mass transfer driven by gravitational radiation (de Miguel et al. 2018). However, the orbital periods of both HM Cnc and V407 Vul decrease (Esposito et al. 2014, and references therein). This was shown to be possible if the donors in these stars still have significant surface hydrogen layers as a result of the prior evolution (D'Antona et al. 2006; Kaplan et al. 2012). There is indeed some evidence that hydrogen contributes to the emission line spectrum of HM Cnc (Roelofs et al. 2010). ES Cet may differ from these systems and is possibly more representative of the bulk of AM CVn stars.

Depending upon the size of the accretor in a massexchanging binary, it is possible that a disc is unable to form, and instead the mass is transferred ploughs directly into the accretor. This is familiar for main-sequence stars in the form of Algol binary stars. The same only becomes possible for typical white dwarfs at periods below 10 min (e.g., Marsh \& Steeghs 2002; Roelofs et al. 2010). Such direct impact is likely to lead to strong asymmetries visible in both photometry and spectroscopy. On the other hand, if a disc forms, it tends to be relatively axisymmetric, albeit with a disturbance caused by the gas stream at the edge of the disc. In the case of discs, we see broad absorption lines in some high-state systems or double-peaked line emission from the disc plus a feature associated with the gas
Table 1. Log of ES Cet observations.

\begin{tabular}{llccl}
\hline \hline Telescope & Date & $\begin{array}{c}\text { Exp. } \\
(\mathrm{s})\end{array}$ & UT interval & $\begin{array}{l}\text { No. of } \\
\text { frames }\end{array}$ \\
\hline Magellan & 27 Oct 2002 & $30-40$ & $03: 04-08: 04$ & 357 \\
& 28 Oct 2002 & $30-60$ & $04: 37-08: 15$ & 171 \\
VLT & 28 Oct 2003 & 60 & $00: 03-08: 23$ & 230 \\
\hline
\end{tabular}

stream/disc impact, which executes a sinusoidal radial velocity curve with a full amplitude similar to the separation between the peaks (e.g., Marsh 1999; Morales-Rueda et al. 2003). HM Cnc and V407 Vul both appear to be in a state of direct impact (Barros et al. 2007). The next known AM CVn star, with a longer orbital period than ES Cet, SDSS J135154.46-064309.0 $\left(P_{\text {orb }}=\right.$ 15.7 min, Green et al. 2018), is a disc-accreting system. ES Cet's period is close to the likely dividing line between direct impact and disc accretion and so its mode of accretion is not clear ab initio. Based on an analysis of the light curves, Espaillat et al. (2005) suggested that in fact ES Cet might be in a state of directimpact accretion as well, but this issue has not been settled in the years since.

The issues outlined above motivated us to carry out a spectroscopic study of ES Cet. Spectroscopy, moreover, is a means to confirm the basic hypothesis that the $620 \mathrm{~s}$ photometric period is indeed the binary period. Likely though this seems, given the nature of ES Cet and the way in which its period is evolving, it should certainly be put to the test. The structure of this paper is the following: Sect. 2 contains details about observations. In Sect. 3, we describe the average spectra. Section 4 presents information about the trailed spectra. Doppler maps are presented in Sect. 5. The studies of polarisation and pulsations are reported in Sects. 6 and 7, respectively. The summary and conclusions of our campaign are given in Sect. 8.

\section{Observations}

We acquired spectroscopy of ES Cet during two campaigns in 2002 and 2003. During the first run, 528 spectra were obtained on two nights from 2002 October 27 to 28. The Boller \& Chivens Spectrograph (B\&C) was used to acquire low-resolution spectra on the $6.5 \mathrm{~m}$ Magellan-Clay telescope at Las Campanas Observatory, and covered the range from 3850 to $5500 \AA$. A 0.7 arcsec slit and a 1200 line $\mathrm{mm}^{-1}$ grating yielded a spectral resolution of 2 pixels and a dispersion of $0.80 \AA$ pixel $^{-1}$. A wide-slit exposure of the spectrophotometric standard star LTT377 was used to obtain a nominal count to flux calibration for the spectra.

The second run was conducted on 2003 October 28 using the FORS1 spectrograph on the VLT telescope. This time 230 spectra were obtained with the range from 4300 to $8600 \AA$. A 1.0 arcsec slit and a grism GRIS300V yielded a spectral resolution of 2 pixels and a dispersion of $5.30 \AA$ pixel $^{-1}$ for $2 \times 2$ binning.

Table 1 gives an overview of our observations. All images were bias-subtracted and flat field-corrected. Extraction and calibration were carried out using the software packages PAMELA and MOLLY ${ }^{1}$ (Marsh 1989).

1 http://deneb.astro.warwick.ac.uk/phsaap/software/ 

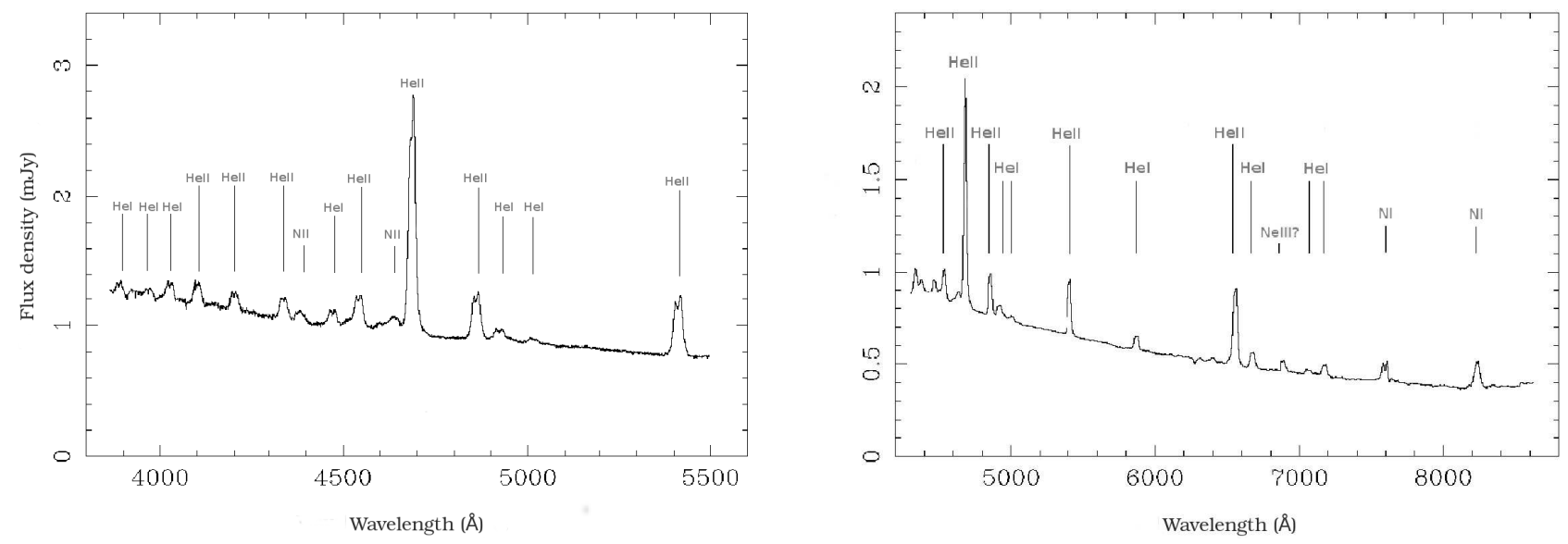

Fig. 1. Mean spectra of ES Cet taken with Magellan 2002 October 27-28 (left panel) and the VLT on 2003 October 28 (right panel). All lines can be identified with HeII, HeI and NI, or NII.

\section{Average spectra}

The average Magellan and VLT spectra of ES Cet are shown in the left and right panels in Fig. 1, respectively. They consist of a blue continuum with a series of strong emission lines. Most of the identifiable features can be interpreted as either neutral (HeI) or ionised (HeII) helium. The lines from neutral helium are weaker than the ionised helium peaks. Neither hydrogen nor metallic lines are detectable, apart from nitrogen. Nitrogen-rich matter is produced in the CNO-cycle, hence nitrogen seems to be abundant in most AM CVn stars (e.g., Marsh et al. 1991; Roelofs et al. 2009; Carter et al. 2014a; Kupfer et al. 2016).

To test for the presence of hydrogen in ES Cet, we plotted the $6560 \AA$ line as a function of velocity two times over, firstly relative to the central wavelength corresponding to the HeII line, and secondly for the $\mathrm{H} \alpha$ line. We compare these to the velocity profile of HeII 4686 in Fig. 2. It is evident that the profiles are better aligned on the assumption that the 6560 line also comes from ionised helium rather than hydrogen. There is thus no evidence for hydrogen in ES Cet. We also measured the radial velocities of ES Cet from the HeII 4685.75 line, and from the wavelength corresponding to the $6560.10 \mathrm{HeII}$ line and to the $6562.72 \mathrm{H} \alpha$ line. The mean difference between the 6560.10-4685.75 lines is $-56.1 \pm 1.8 \mathrm{~km} \mathrm{~s}^{-1}$, while for the $6562.72-4685.75$ case this would be $175.9 \pm 1.8 \mathrm{~km} \mathrm{~s}^{-1}$. Neither is consistent with zero (not surprising because there are some profile differences), but the HeII identification is clearly favoured.

Our measurements of line fluxes and equivalent widths are given in Table 2. The majority of these spectral lines have a broad, double-peaked profile, which is clearly seen in the spectra of ES Cet; for example, in the HeII 5411 line. This is a key feature of binary systems with accretion. In some AM CVn stars, triple-peaked emission lines are observed, including GP Com (Marsh 1999), SDSS J120841.96+355025.2, SDSS J152509.57+360054.50 and SDSS J012940.05+384210.4 (Kupfer et al. 2013), SDSS J113732.32+405458.3, and SDSS J150551.58+065948.7 (Carter et al. 2014b). Smak (1975) and Nather et al. (1981) suggested that the lines are made up of a double-peaked profile from an accretion disc, and a separate narrow component near the centre of the line known as a 'central spike'. This feature is thought to originate close to the surface of the accreting white dwarf (Marsh 1999; Morales-Rueda et al. 2003). There is no evidence for the 'central spike' feature in any of ES Cet's emission lines.

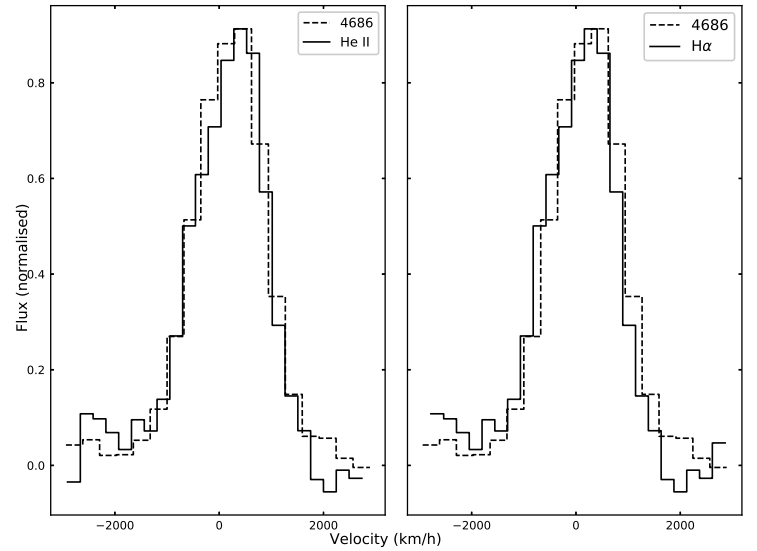

Fig. 2. Emission lines of ES Cet at $4686 \AA$ (dotted line) and $6560 \AA$ (black line). The $6560 \AA$ line was converted into velocity space treated as a HeII line with a central wavelength of $6560.10 \AA$ (left panel), and as a $\mathrm{H} \alpha$ line with a central wavelength of $6562.72 \AA$ (right panel). Both lines are shifted, but the discrepancy is more significant for the $\mathrm{H} \alpha$ case (see Sect. 3).

\section{Trailed spectra}

We present the phased-binned trailed spectra for the strongest HeII and HeI lines of ES Cet on the upper panels in Figs. 3 and 4. Twenty phase bins were equally spaced around the cycle, and we display one cycle repeated twice for clarity. The time-resolved spectra were folded on the ephemeris given by Copperwheat et al. (2011), which spans the epochs of our spectra:

$\mathrm{BMJD}(\mathrm{TDB})=52200.980575(6)+0.00717837598(3) E$,

where $E$ is the cycle number. The trailed spectra show clear evidence of double-peaked emission lines with a sinusoidal Swave component superposed in most instances. Therefore, these data confirm that the $620 \mathrm{~s}$ photometric period is indeed orbital, beyond any doubt. To confirm this quantitatively, we measured radial velocities and computed the Lomb-Scargle periodogram corresponding to the strong HeII 4686 line (Fig. 6). A clear signal is seen at 139 cycles per day. A weaker group of peaks appears at two times this frequency. Zooming in on the main signal, one can see that the strongest peak occurs at $f=139.306725$ cycles per day ( $P_{\text {orb }}=0.007178404$ days $)$, with the two nextstrongest peaks occurring at the usual \pm 1 cycle per day aliases. 
Table 2. Mean fluxes and equivalent widths of lines of ES Cet.

\begin{tabular}{|c|c|c|c|c|c|}
\hline \multicolumn{3}{|c|}{ Magellan } & \multicolumn{3}{|c|}{ VLT } \\
\hline Line & $\begin{array}{c}\text { Flux } \\
\left(10^{-15} \mathrm{ergs} \mathrm{s}^{-1}\right)\end{array}$ & $\begin{array}{l}\text { EW } \\
(\AA)\end{array}$ & Line & $\begin{array}{c}\text { Flux } \\
\left(10^{-15} \mathrm{ergs} \mathrm{s}^{-1}\right)\end{array}$ & $\begin{array}{l}\text { EW } \\
(\AA) \\
\end{array}$ \\
\hline HeI 3888 & $2.6 \pm 0.1$ & $1.12 \pm 0.05$ & HeII 4541 & $9.5 \pm 0.2$ & $6.50 \pm 0.10$ \\
\hline HeI 3964 & $3.8 \pm 0.1$ & $1.53 \pm 0.05$ & HeII 4686 & $42.2 \pm 0.2$ & $38.2 \pm 0.1$ \\
\hline HeI 4026 & $5.7 \pm 0.1$ & $2.55 \pm 0.05$ & HeII 4860 & $8.5 \pm 0.1$ & $8.74 \pm 0.05$ \\
\hline HeII 4100 & $6.7 \pm 0.1$ & $3.20 \pm 0.05$ & HeI 4921 & $3.1 \pm 0.1$ & $3.32 \pm 0.05$ \\
\hline HeII 4200 & $5.1 \pm 0.1$ & $2.66 \pm 0.05$ & HeI 5015 & $0.89 \pm 0.1$ & $1.01 \pm 0.05$ \\
\hline HeII 4339 & $6.6 \pm 0.1$ & $3.99 \pm 0.05$ & HeII 5411 & $9.68 \pm 0.1$ & $14.4 \pm 0.05$ \\
\hline NII 4379 & $3.6 \pm 0.1$ & $2.25 \pm 0.05$ & HeI 5876 & $2.2 \pm 0.1$ & $4.38 \pm 0.05$ \\
\hline HeI 4471 & $5.0 \pm 0.1$ & $3.37 \pm 0.05$ & HeII 6560 & $50.1 \pm 0.2$ & $31.4 \pm 0.1$ \\
\hline HeII 4541 & $12.1 \pm 0.2$ & $8.51 \pm 0.1$ & HeI 6678 & $2.3 \pm 0.05$ & $7.04 \pm 0.1$ \\
\hline NII 4643 & $7.0 \pm 0.2$ & $5.23 \pm 0.1$ & NeIII 6886? & $1.26 \pm 0.05$ & $4.32 \pm 0.1$ \\
\hline HeII 4686 & $55.1 \pm 0.2$ & $43.5 \pm 0.1$ & HeI 7065 & $7.2 \pm 0.2$ & $2.7 \pm 0.1$ \\
\hline HeII 4860 & $11.7 \pm 0.1$ & $10.23 \pm 0.05$ & HeI 7160 & $1.7 \pm 0.2$ & $6.89 \pm 0.2$ \\
\hline HeI 4921 & $3.4 \pm 0.1$ & $3.05 \pm 0.05$ & NI $7608^{(a)}$ & - & - \\
\hline HeI 5015 & $1.3 \pm 0.1$ & $1.25 \pm 0.05$ & NI 8242 & $3.4 \pm 0.2$ & $20.74 \pm 0.2$ \\
\hline HeII 5411 & $13.0 \pm 0.1$ & $16.6 \pm 0.1$ & & & \\
\hline
\end{tabular}

Notes. ${ }^{(a)}$ Line present but could not be measured reliably, due to a blend with another line.

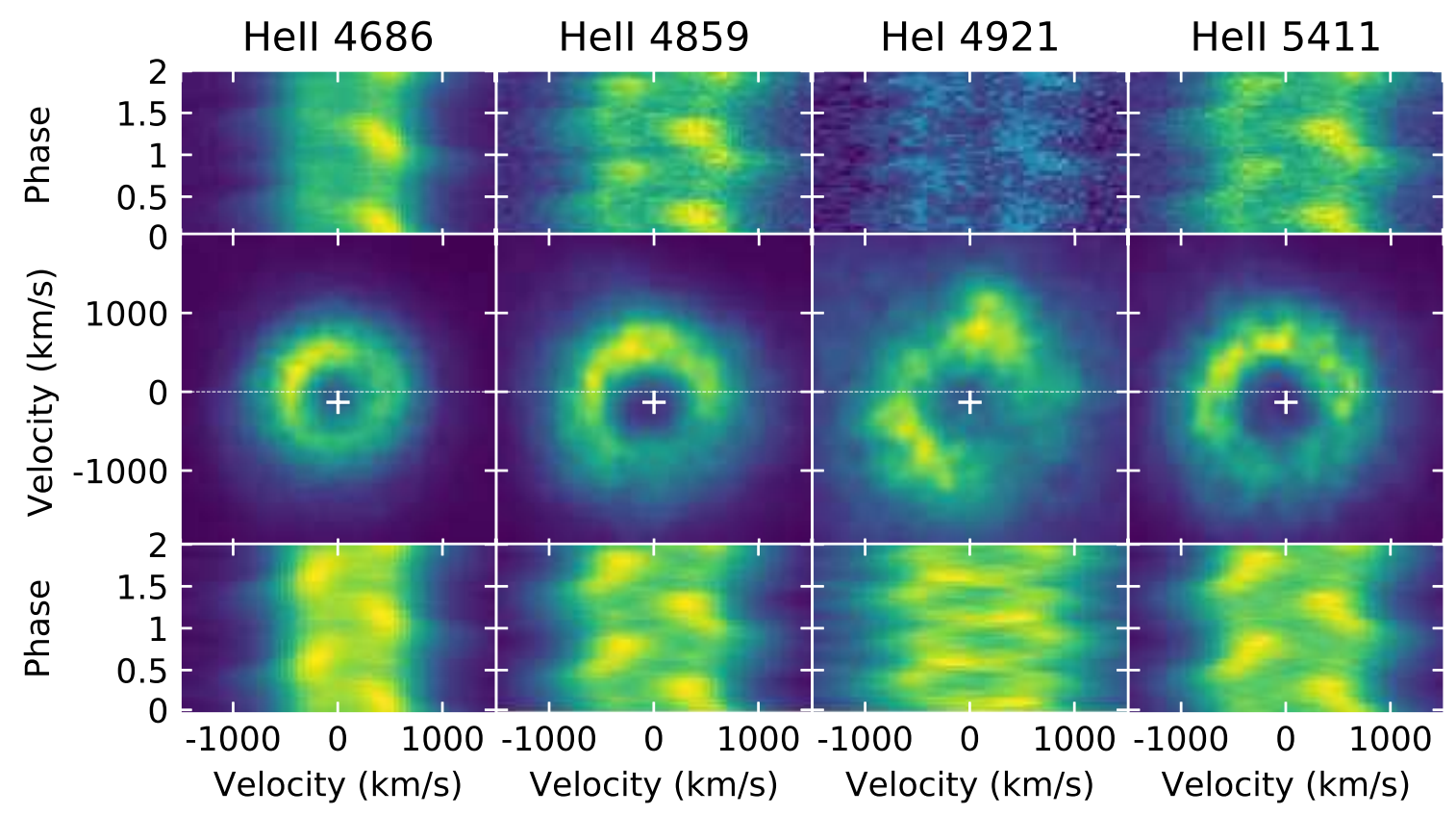

Fig. 3. Top panels: phase-folded, continuum-normalised and subtracted trailed spectra of four of the lines from the Magellan data. Middle and bottom panels: equivalent Doppler maps and fits. White crosses mark the centre of a disc. At phase 1, the HeII 4859 data show a dark feature, especially on the blueshifted side, and slightly delayed in phase near zero velocity, which is the signature of an eclipse of the disc.

The period of the strongest peak corresponds to the one presented by Copperwheat et al. (2011), and hence represents the orbital period of the binary.

In the upper panels of Figs. 3 and 4, one can see a dark, near-horizontal line on the blueshifted side of the HeII 4859, HeII 5411, HeII 4339, and HeII 4541 trailed spectra. HeII 4859 shows it best of all and demonstrates that it extends to the redshifted side of the line, although bright-spot emission dominates on the redshifted side beyond $+400 \mathrm{~km} \mathrm{~s}^{-1}$. The feature, which is shown magnified in Fig. 5 (indicated by the white lines), has all the signs of a 'rotational disturbance' (Greenstein \& Kraft 1959) caused as the disc is eclipsed by the mass donor. For instance, it occurs earliest in phase on the blueshifted peak of the emission line, as expected for a prograde orbiting disc. The feature is seen close to phase zero on the ephemeris of Copperwheat et al. (2011), which is referenced to minimum light. This indicates that the photometric signal is primarily caused by eclipses of the disc. This association is further confirmed by the phasing of the $\mathrm{S}$-wave, which is in a standard location relative to phase zero in the trailed spectra, as also confirmed by the location of the bright emission in the Doppler maps.

Thus, we conclude that ES Cet's outer disc is eclipsed. The eclipse can be traced as far as $\sim-800 \mathrm{~km} \mathrm{~s}^{-1}$ (Fig. 5). Given that the outermost velocity in the disc is around $600 \mathrm{~km} \mathrm{~s}^{-1}$ from the 


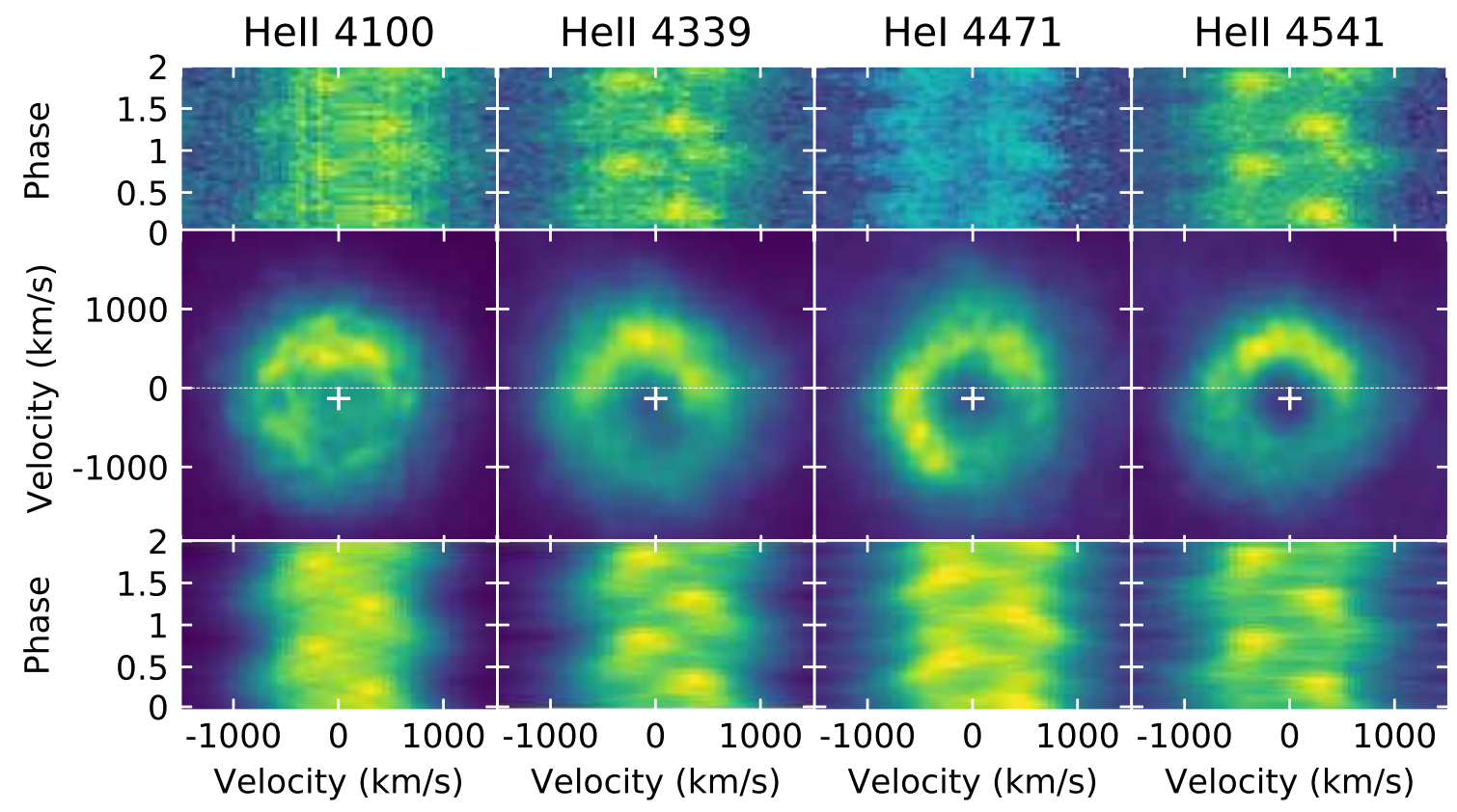

Fig. 4. As in Fig. 3, trailed spectra and the equivalent Doppler maps and fits for four more lines from the Magellan data.
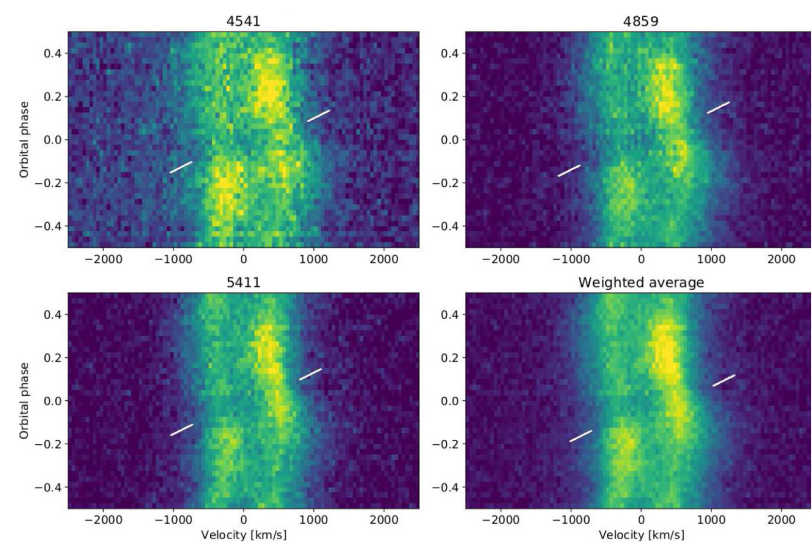

Fig. 5. Closeup of the profiles near eclipse in trailed spectra of three of the lines from the Magellan data: HeII 4541 (top left), HeII 4859 (top right), HeII 5411 (bottom left), and the average of these three lines (bottom right). The eclipse is indicated by the white lines and is visible as a darkening around phase 0.0 that slopes upwards from the blue- to the redshifted sides of the lines.

emission line peak velocity, and that $V \propto R^{-1 / 2}$ in a Keplerian disc, the eclipse therefore reaches a radius in the disc, which is a fraction $(600 / 800)^{2} \approx 50 \%$ of the outer disc radius. The signal-to-noise ratio in the line wings prevents us from determining whether the eclipse reaches the white dwarf itself, although this seems unlikely given the 10 to $20 \%$ depth of the photometric eclipses (Copperwheat et al. 2011). Our spectra had exposure times of $60 \mathrm{~s}$, corresponding to $\sim 0.1$ in terms of orbital phase, very comparable to the vertical extent of the eclipse feature in Fig. 5 at any one velocity in the lines. The data are therefore vertically smeared by this amount, and so shorter exposure spectra have the potential to reveal deeper and sharper variations that may allow the orbital inclination of ES Cet to be pinned down. The eclipse may also encompass the bright spot, as is perhaps best indicated by the HeII 4859 trail in Fig. 5 .

The various trails are similar, but not identical, and HeII 4686 in particular is noticeably asymmetric from red- to blueshifted sides. The $\mathrm{S}$-wave from the bright-spot also seems most visible at its radial velocity extremes. These features may hint at vertical structure and self-obscuration within the disc. This may not be surprising as ES Cet is viewed at high inclination, and it is also a high accretion rate system.

\section{Doppler maps}

To accurately track the phase of the S-wave signal, we back-projected the trailed spectra into a Doppler tomogram (Marsh \& Horne 1988). The method of Doppler tomography enables the projection of a series of phase-resolved spectra on to a two-dimensional map in velocity coordinates (for review: Steeghs 2003; Marsh 2001). In Doppler tomograms, emission features that are not stationary in the binary frame or move on a period different from the orbital period will spread out over the resulting Doppler tomogram, while stationary, emission features add up constructively. Not only is the method of Doppler tomography useful to separate features that move with a different amplitudes and/or phase, but it allows the tracking of asymmetric structures in accretion discs and reveals details of the gas flow in a variety of systems. For analysis of AM CVn systems, Doppler tomography has also proved to be beneficial, for example, to the investigation of the structure of an accretion disc (Breedt et al. 2012), the discovery of multiple bright spots (Roelofs et al. 2006a; Kupfer et al. 2013), or for searching for the presence of central spikes (Kupfer et al. 2016).

For all the trailed spectra, we computed the corresponding Doppler tomograms using the software package DOPPLER ${ }^{2}$ (Marsh \& Horne 1988). The tomograms are presented in the second rows of Figs. 3 and 4 . The bottom panels show trailed spectra computed from the maps. Once again, for each map and trailed spectrum, we used the ephemeris given by Copperwheat et al. (2011). All the Doppler tomograms show a similar structure, with a bright ring corresponding to emission from the accretion disc. In the case of the bright spot, the situation is more complicated. It is located mostly towards the upper

\footnotetext{
2 https://github.com/trmrsh/trm-doppler
} 

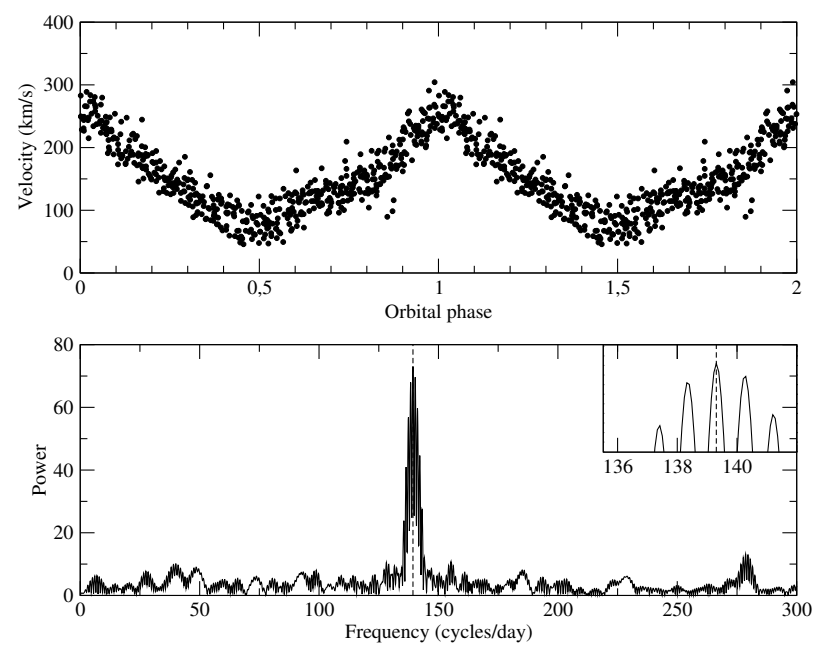

Fig. 6. Top panel: radial velocities of the disc through the S-wave measured from the HeII 4686 line folded on the best fitting period. Bottom panel: Lomb-Scargle periodogram for the same line, with a vertical dashed line showing the position of the ephemeris of Copperwheat et al. (2011). A zoom presents a magnified view of the strongest peak.

left, which, as remarked above, is the expected location, but it has a smeared structure and is not consistent from line to line. Partly this might be azimuthal smearing due to ES Cet's short period and the $60 \mathrm{~s}$ long exposures as discussed earlier for the eclipses; but, in addition, ES Cet clearly is a very high accretion rate system, and the Doppler maps of high accretion rate CVs (nova-likes) have often proved hard to unravel, so we do not attach much significance to this finding. Indeed, compared to many nova-likes, which exhibit single-peaked lines even when deeply eclipsing, ES Cet is well behaved.

\section{Polarimetry}

Given the strong HeII emission in the magnetic polar (AM Her) class of CVs, we decided to obtain spectropolarimetry of ES Cet. We took one night of circular spectropolarimetry using the FORS1 spectrograph of the VLT on 2003 October 28. We took spectra for slightly over eight hours, obtaining 230 exposures of $60 \mathrm{~s}$ each, the remaining time taken up with overheads of rotating the Wollaston prism.

The average polarisation is consistent with zero, with an uncertainty of order $0.1 \%$ (Fig. 7). To check that this could not be the result of cancellation of opposing senses of circular polarisation, we also calculated the power spectrum of the polarisation light curve integrated from 4400 to $7000 \AA$ (Fig. 8). This is also a null result, and in this case we would have expected to detect a semi-amplitude of $0.05 \%$. We illustrate this by injecting such a signal at the expected frequency (dashed line in Fig. 8) that produces a peak of height $\sim 6$, which has a probability (frequency known in advance) of $\exp (-6) \sim 0.25 \%$. We conclude that there is no circular polarisation in ES Cet at a level of 0.05 to $0.1 \%$, and thus no direct sign of magnetic accretion; the mystery of the absence of signs of magnetism amongst AM CVn stars continues.

\section{Discussion}

Several models have been proposed to explain the observed properties of the two shortest period AM CVn stars, HM Cnc and V407 Vul (Ramsay et al. 2000; Israel et al. 2003; Barros et al.

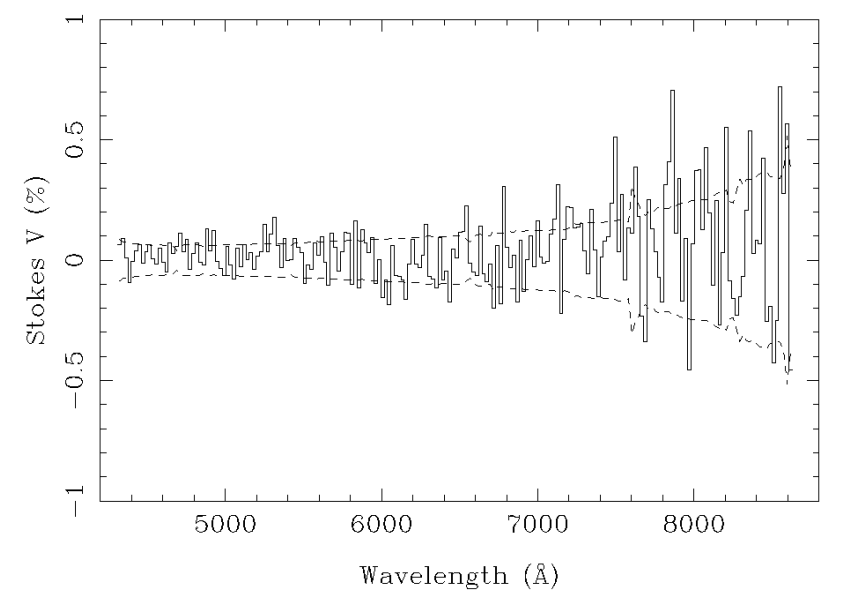

Fig. 7. Mean percentage circular polarisation of ES Cet observed with the VLT on 2003 October 28. The dashed lines show the estimated uncertainties $(1 \sigma)$.

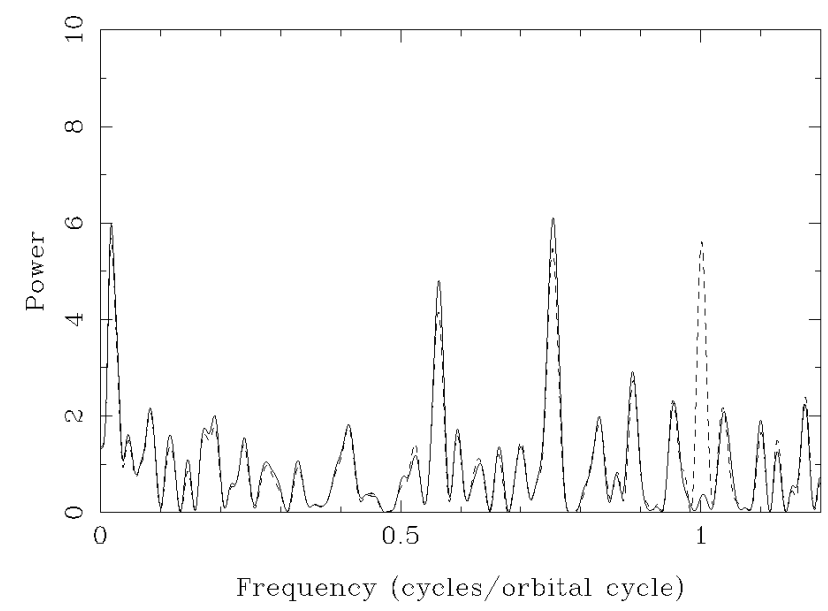

Fig. 8. Lomb-Scargle periodogram of the light curve of the circular polarisation integrated from 4400 to $7000 \AA$. Any signal on the orbital period should appear at 1 cycle/orbital cycle. The dashed line shows the periodogram after the addition of a signal with a semi-amplitude polarisation of $\sim 0.05 \%$ at the expected frequency.

2007). The intermediate polar (IP) model (Motch et al. 1996) holds that these systems are not ultra-compact binaries at all, but rather have orbital periods of several hours, and the ultra-short periods then represent the spins of magnetic white dwarfs. The unipolar induction (UI) model (Wu et al. 2002) is the only model without a Roche lobe filling secondary and is essentially equivalent to the model proposed by Goldreich \& Lynden-Bell (1969) for the Jupiter-Io system. In the direct-impact accretion model (Marsh \& Steeghs 2002), a Roche lobe-filling white dwarf loses its mass to its more massive white dwarf companion, and the accretion stream hits the accretor directly without forming a disc. This is presently the most widely-accepted model of HM Cnc and V407 Vul (Steeghs et al. 2006; Wood 2009; Roelofs et al. 2010). The natures of AM CVn stars with longer orbital periods; for example, SDSS J135154.46-064309.0 $\left(P_{\text {orb }}=15.7\right.$ min, Green et al. 2018) and AM CVn itself $\left(P_{\text {orb }}=17.1 \mathrm{~min}\right.$, Nelemans et al. 2001c), are better established. Their spectra show an absence of hydrogen, the presence of helium lines, many of which are the double-peaked emission (or absorption) lines characteristic of sources accreting via discs. Also, detection of one or even two bright spots are frequently reported 
(Kupfer et al. 2016). Based on the photometric observations, Espaillat et al. (2005) concluded that ES Cet follows the directimpact scenario and is therefore similar to $\mathrm{HM} \mathrm{Cnc}$ and V407 Vul. However, we find instead clear evidence of an accretion disc along with a smeared spot-like structure, placing it very much in the same bracket as the bulk of longer period AM CVn systems.

Although ES Cet has a disc and is more like the other ultra-compact binaries, AM CVn stars at longer periods than ES Cet tend to have very different spectra, with weak absorption lines; for example, HP Lib (Roelofs et al. 2007). AM CVn itself (Roelofs et al. 2006b) shows an absorption spectrum dominated by HeI, with only HeII 4686 in emission, whereas ES Cet is very much an emission line system without any presence of absorption lines. This suggests a difference in the vertical temperature structure within the disc. That is, in AM CVn one sees light from a region where the temperature drops along the line of sight towards the observer, whereas in ES Cet it increases. The high orbital inclination implied by the eclipses may also play a role. Support for this comes from the discovery of Burdge et al. (2020) of ZFT J1905+3134, an eclipsing AM CVn system with a period of $\sim 17.2 \mathrm{~min}$, which also shows strong HeII emission features similar to ES Cet.

\section{Conclusions}

We have described results of Magellan and VLT spectroscopic and spectropolarimetric surveys aimed at understanding the internal accretion structure of the helium-rich binary ES Cet. Time-resolved spectra reveal strong variability in the emission lines on the $620 \mathrm{~s}$ period found from photometry. Double-peaked emission is clearly visible in the lines in our spectra, as is characteristic of an accretion disc. This confirms that the photometric period of $620 \mathrm{~s}$ first reported by Warner \& Woudt (2002) is ES Cet's orbital period. We find no periodic signals on frequencies unrelated to the $620 \mathrm{~s}$ orbital period.

Due to the strong HeII emission in magnetic CVs, which is also seen in ES Cet, we conducted one night of circular spectropolarimetry, but we found no circular polarisation placing an upper limit of $0.1 \%$. The strength of HeII in ES Cet more likely reflects its high accretion rate and state of excitation.

We discovered a short-lived, phase-dependent flux deficit in the trailed spectra of the HeII 4859, HeII 5411, HeII 4339, and HeII 4541 lines, consistent with a 'rotational disturbance' (Greenstein \& Kraft 1959). This shows that the outermost parts of the disc in ES Cet are eclipsed by the mass donor. The timing of the deficit is consistent with the phase of photometric minimum light, indicating that a significant part of the orbital light curve may be caused by eclipses. The eclipse extends over about half the outer disc in radius, but probably does not reach the white dwarf itself given the relatively shallow photometric eclipse. Higher time resolution spectroscopy of ES Cet could refine the exact radial extent of the eclipse and therefore constrain the orbital inclination.

For all the trailed spectra, we computed the corresponding Doppler tomograms. All the Doppler tomograms show evidence for a similar structure, with a bright ring corresponding to emission from the accretion disc. Several also show a brightness maximum at the expected location of the gas stream or disc impact ('bright spot').

We conclude that, with the third shortest orbital period of any AM CVn star $\left(P_{\text {orb }}=620 \mathrm{~s}\right)$, ES Cet is the system with the shortest orbital period that hosts an accretion disc, and that the disc is eclipsed.
Acknowledgements. Project was supported by Polish National Science Center grants awarded by decisions: DEC-2015/16/T/ST9/00174 for KB. TRM and DS acknowledge support from the Science and Technology Facilities Council (STFC) grant numbers ST/P000495/1 and ST/T000406/1. Based on observations collected at the European Organisation for Astronomical Research in the Southern Hemisphere under ESO programme 072.D-0119(A) as well as data gathered with the $6.5 \mathrm{~m}$ Magellan Telescopes located at Las Campanas Observatory, Chile.

\section{References}

Barros, S. C. C., Marsh, T. R., Dhillon, V. S., et al. 2007, MNRAS, 374, 1334 Breedt, E., Gänsicke, B. T., Marsh, T. R., et al. 2012, MNRAS, 425, 2548 Brooks, J., Bildsten, L., Marchant, P., \& Paxton, B. 2015, ApJ, 807, 78 Brown, W. R., Kilic, M., Hermes, J. J., et al. 2011, ApJ, 737, L23 Burdge, K. B., Prince, T. A., Fuller, J., et al. 2020, ApJ, 905, 32 Carter, P. J., Steeghs, D., Marsh, T. R., et al. 2014a, MNRAS, 437, 2894 Carter, P. J., Gänsicke, B. T., Steeghs, D., et al. 2014b, MNRAS, 439, 2848 Copperwheat, C. M., Marsh, T. R., Dhillon, V. S., et al. 2011, MNRAS, 413, 3068

D’Antona, F., Ventura, P., Burderi, L., \& Teodorescu, A. 2006, ApJ, 653, 1429 de Miguel, E., Patterson, J., Kemp, J., et al. 2018, ApJ, 852, 19

Downes, R. A., \& Shara, M. M. 1993, PASP, 105, 127

Espaillat, C., Patterson, J., Warner, B., et al. 2005, PASP, 117, 189 Esposito, P., Israel, G. L., Dall'Osso, S., \& Covino, S. 2014, A\&A, 561, A117 Green, M. J., Hermes, J. J., Marsh, T. R., et al. 2018, MNRAS, 477, 5646 Green, M. J., Marsh, T. R., Steeghs, D., et al. 2019, MNRAS, 485, 1947 Greenstein, J. L., \& Kraft, R. P. 1959, ApJ, 130, 99

Gilfanov, M., \& Bogdán, Á. 2010, Nature, 463, 924 Goldreich, P., \& Lynden-Bell, D. 1969, ApJ, 156, 59 Haberl, F., \& Motch, C. 1995, A\&A, 297, L37

Israel, G. L., Covino, S., Stella, L., et al. 2003, ApJ, 598, 492 Kaplan, D. L., Bildsten, L., \& Steinfadt, J. D. R. 2012, ApJ, 758, 64 Kupfer, T., Groot, P. J., Levitan, D., et al. 2013, MNRAS, 432, 2048 Kupfer, T., Steeghs, D., Groot, P. J., et al. 2016, MNRAS, 457, 1828 Kupfer, T., Korol, V., Shah, S., et al. 2018, MNRAS, 480, 302

Marsh, T. R. 1989, PASP, 101, 1032

Marsh, T. R. 1999, MNRAS, 304, 443

Marsh, T. R. 2001, in Lecture Notes in Physics, eds. H. M. J. Boffin, D. Steeghs, \& J. Cuypers (Berlin: Springer-Verlag), 573, 1

Marsh, T. R., \& Horne, K. 1988, MNRAS, 235, 269

Marsh, T. R., \& Steeghs, D. 2002, MNRAS, 331, L7

Marsh, T. R., Horne, K., \& Rosen, S. 1991, ApJ, 366, 535

Marsh, T. R., Nelemans, G., \& Steeghs, D. 2004, MNRAS, 350, 113

Morales-Rueda, L., Marsh, T. R., Steeghs, D., et al. 2003, A\&A, 405, 249

Motch, C., Haberl, F., Guillout, P., et al. 1996, A\&A, 307, 459

Nather, R. E., Robinson, E. L., \& Stover, R. J. 1981, ApJ, 244, 269

Nelemans, G., Yungelson, L. R., Portegies Zwart, S. F., et al. 2001a, A\&A, 365, 491

Nelemans, G., Portegies Zwart, S. F., Verbunt, F., et al. 2001b, A\&A, 368, 939 Nelemans, G., Steeghs, D., \& Groot, P. J. 2001c, MNRAS, 326, 621 Noguchi, T., Maehara, H., \& Kondo, M. 1980, Tokyo Astron. Obs. Annal., 18, 55

Paczyński, B. 1967, Acta Astron., 17, 287

Pala, A. F., Gänsicke, B. T., Breedt, E., et al. 2020, MNRAS, 494, 3799

Podsiadlowski, P., Han, Z., \& Rappaport, S. 2003, MNRAS, 340, 1214

Ramsay, G., Cropper, M., Wu, K., Mason, K. O., \& Hakala, P. 2000, MNRAS, 311,75

Ramsay, G., Green, M. J., Marsh, T. R., et al. 2018, A\&A, 620, A141

Roelofs, G. H. A., Groot, P. J., Marsh, T. R., et al. 2006a, MNRAS, 365, 1109

Roelofs, G. H. A., Groot, P. J., Marsh, T. R., et al. 2006b, MNRAS, 371, 1231

Roelofs, G. H. A., Groot, P. J., Nelemans, G., et al. 2007, MNRAS, 379, 176

Roelofs, G. H. A., Groot, P. J., Benedict, G. F., et al. 2009, MNRAS, 394, 367

Roelofs, G. H. A., Rau, A., Marsh, T. R., et al. 2010, ApJ, 711, L138

Shen, K. J. 2015, ApJ, 805, L6

Shen, K. J., \& Bildsten, L. 2014, ApJ, 785, 61

Smak, J. 1967, Acta Astron., 17, 255

Smak, J. 1975, Acta Astron., 25, 227

Solheim, J.-E. 2010, PASP, 122, 1133

Steeghs, D. 2003, MNRAS, 344, 448

Steeghs, D., Marsh, T. R., Barros, S. C. C., et al. 2006, ApJ, 649, 382

Warner, B. 1995, Cataclysmic Variable Stars (Cambridge: Cambridge University Press)

Warner, B., \& Woudt, P. A. 2002, PASP, 114, 129

Wood, M. A. 2009, MNRAS, 395, 378

Wu, K., Cropper, M., Ramsay, G., et al. 2002, MNRAS, 331, 221

Yungelson, L. R. 2008, Astron. Lett., 34, 620 